\title{
RECOGNITION OF HANDWRITTEN DIGITS USING RBF NEURAL NETWORK
}

\author{
P P S Subhashini ${ }^{1}$, V V K D V Prasad ${ }^{2}$ \\ Department of ECE, RVR \& JC College of Engineering, Chowdavaram, Guntur, 522019, A.P, India, \\ k_shiva_111@yahoo.co.in,varrevkdvp@rediffmail.com
}

\begin{abstract}
Pattern recognition is required in many fields for different purposes. Methods based on Radial basis function (RBF) neural networks are found to be very successful in pattern classification problems. Training neural network is in general a challenging nonlinear optimization problem. Several algorithms have been proposed for choosing the RBF neural network prototypes and training the network. In this paper RBF neural network using decoupling Kalman filter method is proposed for handwritten digit recognition applications. The efficacy of the proposed method is tested on the handwritten digits of different fonts and found that it is successful in recognizing the digits.
\end{abstract}

Keywords: - Neural network, RBF neural network, Decoupled kalman filter Training, Zoning method

\section{INTRODUCTION}

Character recognition is classified into two categories, off-line character recognition and on-line character recognition [1]. In off-line character recognition the system accepts image as input from the scanner. It is more difficult than on-line character recognition because of unavailability of contextual information and lack of prior knowledge like text position, size of text, order of strokes, start point and stop point. Noise will also exist in the images acquired in off-line character recognition. Machine Printed character recognition comes under this category [2]. On-line character recognition system accepts the moment of pen from the hardware such as graphic tablet, light pen and lot of information is available during the input process such as current position, moment's direction, start points, stop points and stroke orders.e.g. handwritten character recognition [3].

There has been a drastic change in our perspective of concept of communication and connectivity with digital revolution. Biometrics play vital role in dealing with problem of authentication. It is the science of identifying or verifying the identity of a person based on physiological or behavioural characteristics. Physiological characteristics include fingerprints, iris, hand geometry and facial image [4]. The behavioural characteristics are actions carried out by a person in a characteristic way and include recognition of signature, machine printed characters, handwriting, and voice. There have been attempts to explore the possibility of efficient manmachine communication through hand written characters. Pattern recognition techniques using RBF neural network are helpful in classification of hand written characters of different users [5].
This paper focuses on handwritten digits recognition using RBF neural network based on decoupling Kalman filter training method. From the results it is found that the proposed method has very high success rate in handwritten digit recognition.

\section{RBF NEURAL NETWORKS}

Radial basis function network (RBF) is a type of artificial network for applications to problems of supervised learning e.g. regression, classification and time series prediction. RBF networks can be used to solve classification problems [6]. The classification problem can be treated as a non-parametric regression problem if the outputs of the estimated function are interpreted as the probability that the input belongs to the corresponding classes.

The training output values are vectors of length equal to the number of classes. After training, the network responds to a new pattern with continuous values in each component of the output vector and these values are interpreted as being proportional to class probability. This network consists of three layers, input layer, hidden layer, output layer as shown in Fig. 1. The $\mathrm{m}$-dimensional input $\mathrm{x}$ is passed directly to a hidden layer. Suppose there are c neurons in the hidden layer, each of the $\mathrm{c}$ neurons in the hidden layer applies an activation function which is a function of the Euclidean distance between the input and an m-dimensional prototype vector [7].

Each hidden neuron contains its own prototype vector as a parameter. The output of each hidden neuron is then weighted and passed to the output layer. The outputs of the network consist of sums of the weighted hidden layer neurons. The design of an RBF requires several decisions that include the 
number of hidden units in the hidden layer, values of the prototypes, the functions used at the hidden units and the weights applied between the hidden layer and the output layer. The performance of an RBF network depends on the number and location (in the input space) of the centres, the shape of the RBF functions at the hidden units and the method used for determining the network weights. Some researchers have trained RBF networks by selecting the centers randomly from the training data. Others have used unsupervised procedures (such as the k-means algorithm) for selecting the RBF centers. Still others have used supervised procedures for selecting the RBF centers.

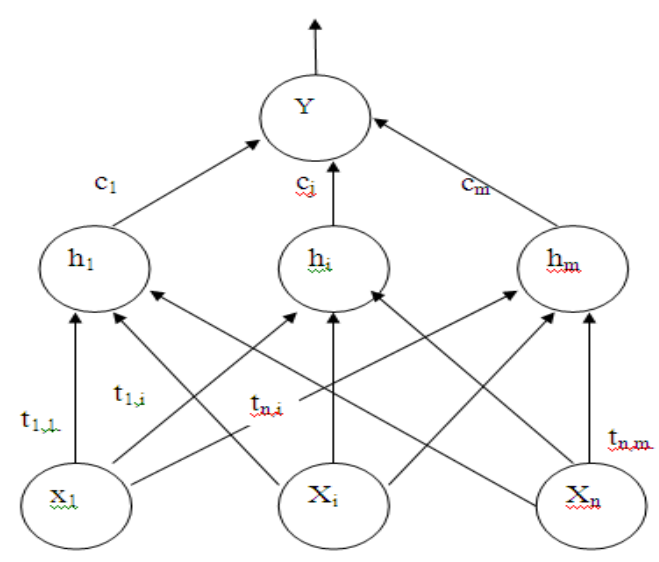

Fig 1 RBF Neural network architecture

\subsection{Working Principle}

The principle of radial basis functions derives from the theory of functional approximation. Given $N_{\text {pairs }}\left(x_{i}, y_{i}\right)$, one looks for a function $f$ of the form: $f(x)=\sum_{i=1}^{k} c_{i} h\left(\left|x-t_{i}\right|\right)$ this, $h$ is the radial basis function (normally a Gaussian function) and $t_{i}$ are the $k$ centers which have to be selected. The coefficients $c_{i}$ are also unknown at the moment and have to be computed. $x_{i}$ and $t_{i}$ are elements of an $\mathrm{n}$-dimensional vector space.

The hidden units compute the Euclidean distance between the input pattern and the vector, which is represented by the links leading to this unit. The activation of the hidden units is computed by applying the Euclidean distance to the function $h$ as $h(x)=e\left(x-c_{i}\right)^{2} / r^{2}$. The parameters for the function $h$ are its center $c$ and its radius $r$. There have been a number of activation functions that include Gaussian function, Thin plate spline function, Multiquadric function and so on for the hidden layer of RBF neural network. In this paper the following function is considered.

$$
g(v)=\left(g_{0}(v)\right)^{1 /(1-p)}
$$

where $p$ is a parameter and $g_{0}(v)$ is a linear function of the form

$$
g_{0}(v)=a v+b
$$

where $a>0$ and $b \geq 0$. The single output neuron gets its input from all hidden neurons. The links leading to the output neuron hold the coefficients ci. The activation of the output neuron is determined by the weighted sum of its inputs. An RBF network is considered non-linear if the basis functions can move or change size or if there is more than one hidden layer otherwise the RBF network is considered linear. The above architecture can easily be extended to include more than one output node depending on the problem that the RBF network is to solve e.g classification into different classes would need as many output nodes as the number of classes.

When the RBF network is used in classification, the hidden layer performs clustering while the output layer performs classification. The hidden units would have the strongest impulse when the input patterns are closed to the centers of the hidden units and gradually weaker impulse as the input patterns moved away from the centers. The output layer would linearly combine all the outputs of the hidden layer. Each output node would then give an output value, which represents the probability that the input pattern falls under that class.

\section{DECOUPLED KALMAN FILTER}

For a large RBF network, the computational expense of the Kalman filter could be burdensome [8]. In fact, the computational expense of the Kalman filter is on the order of $\mathrm{AB} 2$, where $\mathrm{A}$ is the dimension of the output of the dynamic system and $B$ is the number of parameters. In our case, there are $\mathrm{nM}$ outputs and $\mathrm{n}(\mathrm{c}+1)+\mathrm{mc}$ parameters, where $\mathrm{n}$ is the dimension of the RBF output, $\mathrm{M}$ is the number of training samples, $\mathrm{c}$ is the number of prototypes, and $\mathrm{m}$ is the dimension of the RBF input. Therefore, the computational expense of the Kalman filter is on the order of $\mathrm{nM}[\mathrm{n}(\mathrm{c}+1)+\mathrm{mc}] 2$.

The Kalman filter parameter vector can be decoupled by assuming that certain parameter groups interact with each other only at a second-order level. For instance, the Hk matrix contains a lot of zeros, showing that the interaction between various parameter groups can be neglected. In particular, the Hk matrix consists of $n+1$ decoupled blocks. These $n+1$ blocks correspond to the $\mathrm{n}$ sets of $\mathrm{w}$ weights that a4ect the $\mathrm{n}$ output components, and the set of prototypes. This is intuitive because, for example, the $c+1$ weights that impinge on the first component of the output are completely independent of the $c+1$ 
weights that impinge on the second component of the output . Let us use the notation that _ik refers to the ith group (out of $\mathrm{n}+1$ total) of parameters estimated at time step $\mathrm{k}$. Then we have

$\theta 1 \mathrm{k}=\mathrm{w} 1 \ldots \theta \mathrm{nk}=\mathrm{wn}$

$\theta \mathrm{n}+1 \mathrm{k}=[\mathrm{v} 1 \mathrm{~T} \ldots \ldots \mathrm{VcT}] \mathrm{T}$

We will use the notation that Hi corresponding to the i th group of parameters.

$\mathrm{H} 1 \mathrm{k}=\mathrm{H} . . . \mathrm{Hnk}=\mathrm{HHn}+1 \mathrm{k}=\mathrm{Hv}$

where $\mathrm{H}$ (with no subscript) is the $(\mathrm{c}+1) \mathrm{X}$ m matrix . We will use the notation that ykt refers to the element of the target output of the RBF network that are effected by the $i$ th group of parameters

$\mathrm{yk} 1=[\mathrm{y} 11 \ldots \ldots \ldots . \mathrm{y} 1 \mathrm{~m}] \mathrm{T}$

$\mathrm{ykn}=[\mathrm{yn} 1 \ldots \ldots \ldots . . . \mathrm{ynm}] \mathrm{T}$

$\mathrm{ykn}+1=[\mathrm{yn} 1 \ldots \ldots \mathrm{y} 1 \mathrm{~m} \ldots \ldots \mathrm{y} 1 \mathrm{n} \ldots \ldots . \mathrm{ynm}] \mathrm{T}$

Similarly, we use the notation that ht $(\theta \mathrm{k}-1)$ refers to the elements of the actual output of the RBF network that are effected by the $i$ th group of parameters

$\mathrm{h} 1(\theta \mathrm{k}-1)=[\tilde{y} 11 \ldots \ldots . . . \tilde{y} 1 \mathrm{M}] \mathrm{kT}$

$\mathrm{hn}(\theta \mathrm{k}-1)=[\tilde{y} \mathrm{n} 1 \ldots \ldots . . . \mathrm{y} n M] \mathrm{kT}$

$\mathrm{hk}+1(\theta \mathrm{k}-1)=[\tilde{y} 11 \ldots \ldots . . \mathrm{y} 1 \mathrm{M} \ldots$ ỹn $1 \ldots \ldots . . . \mathrm{y} n \mathrm{M}] \mathrm{kT}$

The decoupled Kalman recursion for the ith parameter group is then given by

$\theta$-tk $=\mathrm{f}(\theta-\mathrm{tk}-1)+\mathrm{Kkt}[\mathrm{ykt}-\mathrm{ht}(\theta-\mathrm{tk}-1)]$

$\mathrm{Ki}=$ PktHkt $(\mathrm{Rt}+(\mathrm{Hkt}) \mathrm{T}$ PktHkt)-1

$\mathrm{Pk}+1 \mathrm{t}=\mathrm{Fk}(\mathrm{Pk}-\mathrm{Kkt}(\mathrm{Hkt}) \mathrm{TPkt}) \mathrm{FkT}+\mathrm{QT}$

As before, $f($.$) is the identity mapping and Fk is the identity$ matrix. The above recursion executes $n+1$ times. The first $n$ times, the recursion consists of $\mathrm{M}$ outputs and $(\mathrm{c}+1)$ parameters. The last time, the recursion consists of $\mathrm{nM}$ outputs and $\mathrm{mc}$ parameters. So the computational expense of the Kalman filter has been reduced to the order of $\mathrm{nM}[(\mathrm{c}+1) 2+$ (mc)2]. The ratio of the computational expense of

Standard KF Expense $\quad \mathrm{n} 2(\mathrm{c}+1) 2+\mathrm{m} 2 \mathrm{c} 2+\mathrm{n}(\mathrm{c}$ $+1) \mathrm{mc}$

Decoupled KF Expens $=1) 2+m 2 c 2:(42)$

The computational savings will be most significant for large problems, i.e., problem where $\mathrm{n}$ (the dimension of the output) is large, $\mathrm{m}$ (the dimension of the input) is large, or $\mathrm{c}$ (the number of prototypes) is large. Note that this complexity analysis applies only to the Kalman recursion and does not include the computational expense required for the calculation of the partial derivatives. Also note that for both the standard and decoupled Kalman filters, the computational expense increases linearly with $\mathrm{M}$ (the number of training samples).

\section{EXPERIMENTAL RESULTS}

The results obtained on using the proposed RBF neural network based on decoupling kalman filter training method for recognition of handwritten digits of different fonts are presented in this section. Hand written digits from 0 to 9 of 25 different fonts are taken and they are recognized by using this method. For each hand written digit, after obtaining the thinned image, 16 features are extracted using Zoning method. These features are used to train RBF neural network [9].

The different steps involved in recognition of the digits using this method are shown in the block diagram in the Fig.2

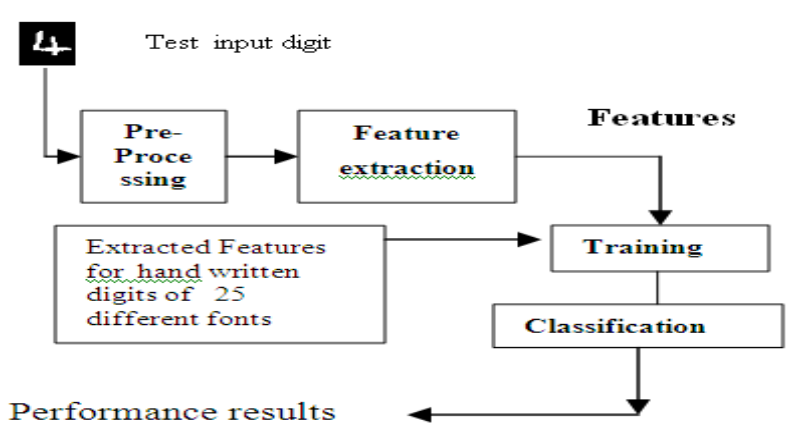

Fig. 2. Block Diagram of Handwritten digit recognition

The RBF networks are trained using the hidden layer function of Eq. (1) The exponential parameter $p$ in Eq. (1) is taken as 2 in the results presented in this section. The training algorithms are initialized with prototype vectors randomly selected from the input data and with the weight matrix $W$ set to zero[10]. From the experiments, it is found that Decoupled Kalman Filter Training algorithm is terminated when the error decreases by less than 0.1 percent. The performance of each of the training methods is explored by averaging its performance over five trials, where each trial consists of a random selection of training and test data. The number of hidden units in the RBF network is varied between 1 and 15 [11].

Fig. 3 shows the number of iterations required for convergence. Percentage of correct classification for number of hidden units is given in Fig.4 for decoupling Kalman filter training [12]. The RBF network is trained with 13 different fonts of each digit and is also tested with 12 other fonts which are different from the trained fonts. It is observed that this method is successful in recognizing the digits of all these 25 fonts.

For space consideration, the results of six hand written digits viz 0 to 5 are only shown here. The features of these handwritten digits are extracted and tabulated in Table 1 and 2. 
Each digit has 16 features. The combination of all these features is called a feature vector. The feature vector is applied to neural network. If the digit is recognized, then the output node corresponding to the digit is one and the remaining are zeros. The training method here applied is supervised training method. This requires the target vector that is the desired output, which is tabulated in Table 3.

Table 1: Features of Handwritten Digits 0 to 5

\begin{tabular}{|c|r|r|r|r|r|r|r|r|}
\hline Hand Written Digit & \multicolumn{7}{|c|}{ Features of Handwritten Digits (8 features) } \\
\hline 0 & 0.526316 & 0.526316 & 0.526316 & 0.526316 & 0 & 1.105263 & 1 & 0 \\
\hline 1 & 0 & 0 & 0 & 0 & 0 & 1.842105 & 0 & 0 \\
\hline 2 & 0 & 0 & 0 & 0 & 0 & 1.157895 & 0.631579 & 0 \\
\hline 3 & 0 & 0 & 0 & 0 & 0 & 1.842105 & 1.315789 & 0 \\
\hline 4 & 0 & 0.052632 & 0 & 0 & 0 & 1 & 1.526316 & 0 \\
\hline 5 & 0 & 0 & 0 & 0 & 0 & 0 & 1 & 0.052632 \\
\hline
\end{tabular}

Table 2: Features of Handwritten Digits 0 to 5

\begin{tabular}{|c|r|r|r|r|r|r|r|r|}
\hline Hand Written Digit & \multicolumn{7}{|c|}{ Features of Handwritten Digits (8 features) } \\
\hline 0 & 0 & 1.421053 & 1.105263 & 0 & 0 & 0 & 0 & 0 \\
\hline 1 & 0 & 0.842105 & 0 & 0 & 0 & 0 & 0 & 0 \\
\hline 2 & 0 & 1.684211 & 1.157895 & 0.105263 & 0 & 0 & 0 & 0 \\
\hline 3 & 0 & 0.894737 & 0.842105 & 0 & 0 & 0 & 0 & 0 \\
\hline 4 & 0 & 0.631579 & 2 & 0.105263 & 0 & 0 & 0 & 0 \\
\hline 5 & 0 & 0 & 1.210526 & 0 & 0 & 0.105263 & 0.421053 & 0 \\
\hline
\end{tabular}

Table 3: Target vector for Hand written digits 0 to 5

\begin{tabular}{|c|c|c|c|c|c|c|}
\hline Node & $\begin{array}{c}\text { Output } \\
\text { node1 }\end{array}$ & $\begin{array}{c}\text { Output } \\
\text { node2 }\end{array}$ & $\begin{array}{c}\text { Output } \\
\text { node3 }\end{array}$ & $\begin{array}{c}\text { Output } \\
\text { node4 }\end{array}$ & $\begin{array}{c}\text { Output } \\
\text { node5 }\end{array}$ & $\begin{array}{c}\text { Output } \\
\text { node6 }\end{array}$ \\
\hline 0 & 1 & 0 & 0 & 0 & 0 & 0 \\
\hline 1 & 0 & 1 & 0 & 0 & 0 & 0 \\
\hline 2 & 0 & 0 & 1 & 0 & 0 & 0 \\
\hline 3 & 0 & 0 & 0 & 1 & 0 & 0 \\
\hline 4 & 0 & 0 & 0 & 0 & 1 & 0 \\
\hline 5 & 0 & 0 & 0 & 0 & 0 & 1 \\
\hline
\end{tabular}




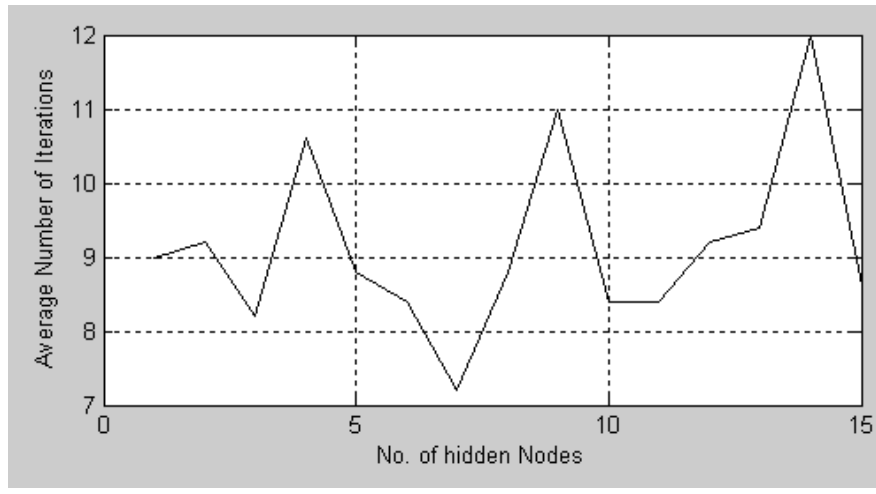

Fig 3. No.of iterations required for No. of hidden nodes

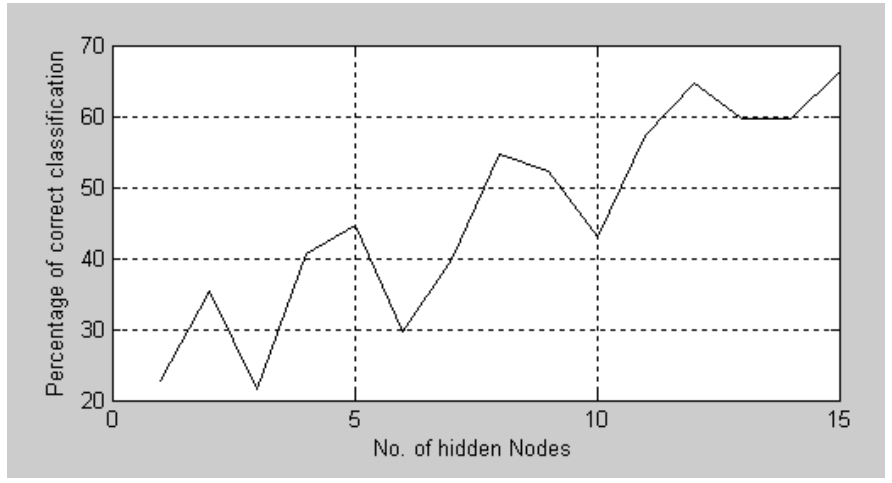

Fig 4. Percentage of Correct Classification for No of Hidden Nodes

\section{CONCLUSIONS}

The RBF Neural Network using decoupled Kalman filter training method is proposed for recognition of handwritten digits of different fonts. The proposed method is tested on handwritten digits of 0 to 9 of 25 different fonts. It is found that this method has very high success rate in recognizing the handwritten digits. This method can be extended to recognize handwritten characters also.

\section{ACKNOWLEDGMENTS}

The authors place on record their thanks to the authorities of RVR\&JC College of Engineering, Guntur, A.P., India for the facilities provided.

\section{REFERENCES}

[1] Naveed Bin Rais , M. Shehzad Hanif and rmtiaz A. Taj, Adaptive Thresholding Technique for Document Image Anaysis, IEEE Trans. on Systems, Man and Cybernetics, Vol.8, 1978, pp.60-66.

[2] Sittisak Rodtook and Yuttapong Rangsanseri, Adaptive Thresholding of Document Images on Laplacian Sign, IEEE Transactions on Pattern analysis and Machine Intelligence, vol.17, No.24, January, 2001.

[3] Jaekyu Ha \& Robert M. Haralick Ihsin, T. Phillips, Document Page Decomposition by the Bounding-Box Projection Technique, IEEE Transactions on Systems, Man, and Cybernetics, Vol.18, No.1, January 1995, pp.1118-1122.

[4] Rangachar Kasturi, Lawrence O'Gorman and Venu Govindaraju, Document analysis: A primer, Sadhana Vol.27, Part 1, February 2002, pp. 3-22.

[5] Jaekyu Ha \& Robert M. Haralick Ihsin, T. Phillips, Document Page Decomposition by the Bounding-Box Projection Technique, IEEE Transactions on Systems, Man, and Cybernetics, Vol.18, No.1, pp.1118-1122, January 1995.

[6] Brijesh K. Verma, Handwritten Hindi Character Recognition Using Multilayer Perceptron and RBF Neural Networks, IEEE Trans. On Neural Networks, Vol.21, August 2004, pp. 2111-2115

[7] O.Batsaikhan and Y.P. Singh, Mongolian Character Recognition using Multilayer Perceptron, Proceedings of the $9^{\text {th }}$ International Conference on Neural Information Processing, Vol. 2,2002.

[8] John Wiley \& Sons, - Technology and Engineering, Kalman Filtering and Neural Networks ,07-Apr2004.

[9] Oivind Due Trier, Anil K.Jain and Torfinn Taxt, Feature Extraction methods for Character recognitionA Survey, Pattern Recognition, Vol. 29, No. 4, 1996, pp.641-662.

[10] P. S. P. Wang and Y. Y. Zhang, A fast and flexible thinning algorithm, IEEE Transactions on Computers, Vol.38, No.5, May 1989.

[11] O.Batsaikhan and Y.P. Singh, Mongolian Character Recognition using Multilayer Perceptron, Proceedings of the $9^{\text {th }}$ International Conference on Neural Information Processing, Vol. 2,2002.

[12] Military Communications Conference, 2003. MILCOM '03. 2003 IEEE paper ,A steady state decoupled Kalman filter technique for multiuser detection Year: 2003 , Page(s): 987 - 992 Vol.2 